\title{
INTEGRAL EQUATIONS FORMULATION OF CLASSICAL ELASTICITY*
}

\author{
$\mathrm{BY}$ \\ R. P. KANWAL \\ Pennsylvania State University
}

1. Introduction. The strong analogy between the potential theory and classical elastostatics is rather well known and has been extensively exploited for various boundary value problems [1]-[9]. In this paper we stress the strong analogy between the Stokes flow equations of hydrodynamics and the equations of classical elasticity-statical as well as dynamical. The general integral formulation of Stokes flow problems was initiated by Lorentz and forms the basis for Oseen's treatise on low Reynolds number hydrodynamics [10]. This method has been recently extended by Williams [11]. It can be applied rather effectively to the corresponding boundary value problems of elastostatics and elastodynamics.

In this paper we discuss three cases. The first case is that of finding the displacement field as well as tractions on a rigid obstacle of an arbitrary shape when translated within an unbounded elastic medium. There results a simple set of integral equations to solve. Since the problems of this nature have been solved extensively, we assume that these integral equations have a known solution. We next consider the case when there is a rigid boundary in the neighborhood of the obstacle. This problem again reduces to that of solving a set of integral equations similar to the first set but more complicated in nature. It is shown that the approximate solution of these equations can be carried out completely, provided the solution for the corresponding case of the infinite medium is known.

The rest of the paper deals with the problems of dynamic elasticity. We have recently studied the vibrations of axially symmetric bodies in an unbounded elastic medium by the method of matched asymptotic expansions [12]. In the present paper we prove that the integral equation approach can be effectively employed to study the same problem and furthermore to generalize it to the obstacles of arbitrary shapes. The paper ends with the example of a thin rigid circular disk which is attached to the free surface of an elastic half space and excited by a periodic force along a direction which makes an angle $\alpha$ with its axis of symmetry.

The main advantage of the present approach is that it permits a unified treatment of a large variety of boundary value problems. In many cases the first order corrections to the known displacement field in an infinite elastic space may be obtained without detailed calculations or the use of the theory of special functions. Furthermore, it is possible to treat obstacles of arbitrary geometries as effectively as it is to treat a body having the spherical symmetry.

2. Elastostatics. The nondimensionalized Navier-Cauchy equations of elastostatics are

$$
(\lambda+\mu) \operatorname{grad} \theta+\mu \nabla^{2} \mathbf{u}=0, \quad \theta=\operatorname{div} \mathbf{u},
$$

*Peceived Al:gust, 4, 1967; revised version received January 12, 1968. 
where $\mathfrak{u}\left(u_{i}, i=1,2,3\right)$ is the displacement vector and $\lambda$ and $\mu$ are Lame's constants of the medium. The displacement vector $\mathbf{u}$ is considered to be a function of Cartesian coordinates $x_{i}$. The above equations have been nondimensionalized by introducing a characteristic geometric length ' $a$ ' in the problem. We can derive integral equations for the solution of Eqs. (1) which are analogous to Green's formulas in potential theory. In order to present these formulas we define certain tensor functions which are the appropriate generalizations of the Green's function of potential theory

Let us consider a light rigid body $B$ and let $S$ be its bounding surface. We further assume that the differential equations (1) have a regular solution in the elastic medium surrounding $B$. We shall designate the domain exterior to $B$ as $D$. Let $P \equiv x_{i}\left(x_{1}, x_{2}, x_{3}\right)$, be any specified point in $D$. To obtain the field caused by the uniform translation of $B$, we calculate the contribution of each elementary point $P^{0}$ at $P$ and then add all these contributions. Let us also keep in mind that, due to the mathematical nature of the differential equations (1), the condition of $\mathbf{u}$ at $\infty$ plays an important role in the solution of these equations. We can now define the above-mentioned tensor function $\mathrm{T}\left(T_{i j}\right)$ as a function of $\mathbf{r}$ and $\mathbf{r}_{0}$, where $\mathbf{r}$ and $\mathbf{r}_{0}$ are the position vectors of the points $P$ and $P^{0}$ respectively

$$
\mathbf{T}=\frac{1}{8 \pi}\left[\mathrm{U} \nabla^{2} \phi-\frac{\lambda+\mu}{\lambda+2 \mu} \operatorname{grad} \operatorname{grad} \phi\right]
$$

or

$$
T_{i j}=\frac{1}{8 \pi}\left[\delta_{i j} \frac{\partial^{2} \phi}{\partial x_{k} \partial x_{k}}-\frac{\lambda+\mu}{\lambda+2 \mu} \frac{\partial^{2} \phi}{\partial x_{i} \partial x_{i}}\right],
$$

where the function $\phi$ is a function of $\left|r-r_{0}\right|$ only and $U=\delta_{i j}$ are the components of the Kronecker delta.

Let us now suppose that $\phi$ satisfies the biharmonic equation

$$
\nabla^{4} \phi=0 .
$$

It then follows by substitution in (1) that

or

$$
(\lambda+\mu) \operatorname{grad} \theta+\mu \nabla^{2} \mathrm{~T}=0,
$$

$$
(\lambda+\mu) \frac{\partial \theta_{i}}{\partial x_{i}}+\mu \frac{\partial^{2} T_{i j}}{\partial x_{k}} \frac{\partial x_{k}}{2}=0
$$

where

$$
\theta_{i}=\partial T_{i k} / \partial x_{k}
$$

This means that for each $i(1,2$ or 3$)$, the vector whose components are $T_{i_{1}}, T_{i 2}, T_{i_{3}}$ and the dilation $\theta_{i}$ constitute solutions of the Eqs. (1). We are thus left with the biharmonic equation (3) to solve.

The quantity $\phi$ is a function of $\left(\mathbf{r}-\mathbf{r}_{0}\right)$ only. Therefore the biharmonic equation (3) written in spherical coordinates becomes

$$
\nabla^{2} \nabla^{2} \phi=0, \quad \nabla^{2}=\frac{1}{r^{2}} \frac{d}{d r}\left(r^{2} \frac{d}{d r}\right)
$$

Since we are looking for such a function $\mathbf{T}$ which tends to zero as $\left|\mathbf{r}-\mathbf{r}_{0}\right|$ tends to infinity 
(to be precise $\mathbf{T}$ is $O(1 / r)$ at $\infty$ ), the solution appropriate to our problem is $\phi=\left(\mathbf{r}-\mathbf{r}_{0}\right)$. This yields

$$
\begin{aligned}
\nabla^{2} \phi & =\frac{2}{\left|\mathbf{r}-\mathbf{r}_{0}\right|} \\
T_{i i} & =\frac{1}{8 \pi}\left[\frac{\lambda+3 \mu}{\lambda+2 \mu} \frac{\delta_{i j}}{\left|r-r_{0}\right|}+\frac{\lambda+\mu}{\lambda+2 \mu} \frac{\left(x_{i}-x_{i}^{0}\right)\left(x_{i}-x_{i}^{0}\right)}{\left|\mathbf{r}-\mathbf{r}_{0}\right|^{3}}\right], \\
\theta_{i} & =-\frac{\mu}{4 \pi(\lambda+2 \mu)} \frac{x_{i}-x_{i}^{0}}{\left|\mathbf{r}-\mathbf{r}_{0}\right|^{3}} .
\end{aligned}
$$

This tensor field $T_{i i}$ was introduced by Somigliana [2] and has appeared in literature thereafter. For instance, it has been recently used by Diaz and Greenberg [13] in connection with the evaluation of the bounds for the solution of the boundary value problems of elasticity.

It is now possible to write down an expression for $\mathbf{u}(P)$, i.e., the solution of Eqs. (1) at an arbitrary point $P$ in the region $D$ by the method which closely resembles the one used in the potential theory. In fact we surround $I I$ with an infinitesimal sphere of radius $r=\sigma$ such that this sphere lies entirely outside $S$. The boundary surface now consists of two parts-the surface $S$ of $B$ and the spherical surface $S_{\sigma}$ given as $r=\sigma$. Let $D_{\sigma}$ denote the volume covered by $S_{\sigma}$. By starting with Green's second identity

$$
\int_{V}\left(f \nabla^{2} g-g \nabla^{2} f\right) d V=\int_{\tilde{S}}\left(f \frac{d g}{d n}-g \frac{d f}{d n}\right) d S,
$$

where $V=D-D_{\sigma}, \bar{S}=S+S_{\sigma}$ and $\mathrm{n}$ is the unit vector drawn outward and normal to $\bar{S}$; setting $f=u_{i}, g=\Psi_{i j}$, it can be shown, in precisely the way it is done in the potential theory, that [2], [14], [16]

$$
\mathbf{u}(P)=-\int_{s}\left[\left\{\mu \frac{d \mathbf{u}}{d n}+(\lambda+\mu) \theta \mathbf{n}\right\} \cdot \mathbf{T}-\mathbf{u} \cdot\left\{\mu \frac{d \mathbf{T}}{d n}+(\lambda+\mu) \theta \mathbf{n}\right\}\right] d S .
$$

By the help of Eq. (4) and Green's theorem, it follows that if $\mathbf{u}$ is constant on $S$, then Eq. (11) reduces to

$$
\mathfrak{u}(P)=-\int_{S} \mathbf{f} \cdot \mathbf{T} d S
$$

where

$$
\mathbf{f}=\mu d \mathbf{u} / d n+(\lambda+\mu) \theta \mathbf{n} .
$$

The above formulas may be applied to the problem of the displacement field generated by the light rigid obstacle $B$ which is embedded in an unbounded elastic space and is given a uniform translation $d_{0}\left(d_{0} / a\right.$ in the nondimensional units). Therefore the boundary conditions are

$$
\mathbf{u}=\left(d_{0} / a\right) \mathbf{I}, \text { on } S, \quad \mathbf{u} \rightarrow 0 \text { as } \mathbf{r} \rightarrow \infty,
$$

where $I$ is the unit direction along the translation of $B$.

Clearly, the displacement field is given by Eq. (12). Furthermore, it follows from the definition of the traction field $t$ that

$$
t_{i}=\lambda \theta n_{i}+\mu n_{i}\left(u_{i, i}+u_{i, i}\right)
$$


and from the definition of the stress tensor that the total static force $\mathbf{F}$ acting on the body $B$ is given by the formula:

$$
F=\int_{S} \mathbf{f} d S
$$

Thus the boundary value problem for the displacement field created in $D$, reduces, by the help of relation (12), to the solution of the integral equation

$$
\frac{d_{0}}{a} \mathbf{I}=-\int \mathbf{f} \cdot \mathbf{T} d S
$$

Since the solutions of boundary value problems of this nature are known for various configurations of $B$, we shall assume that we know the solution of the integral equation (17). With the help of this information we shall proceed to solve more complicated cases.

3. Boundary effects. In this section we consider the first order effects of boundaries on the tractions experienced by a light rigid body which is given a uniform translation. We now have two surfaces $S_{1}$ and $S_{2}$-the surface $S_{1}$ of the displaced body $B$ and the surface $S_{2}$ of the rigid boundary such as a cylinder enclosing $B$. In the region enclosed by any given closed surface $S_{2}$ we define a tensor $T$ which satisfies Eq. (1) with the additional condition that $\mathrm{T}=0$ on $S_{2}$. If both $P$ and $P^{0}$ are within $S_{2}$, then $\mathbf{e} \cdot \mathrm{T}$ may be interpreted as the displacement field at $P$ in the region enclosed by the rigid surface $S_{2}$ when a concentrated force parallel to the unit direction $\mathrm{e}$ is acting at $P^{0}$. Furthermore, $T$ may be written as $T_{1}+T_{2}$, where $T_{1}$ is the Green's tensor defined in the previous section, satisfies Eq. (1) and has Green's function type singularity at infinity:

$$
\mathrm{T}_{1}=\frac{1}{8 \pi}\left(\mathrm{U} \nabla^{2}\left|\mathbf{r}-\mathrm{r}_{0}\right|-\frac{(\lambda+\mu)}{(\lambda+2 \mu)} \operatorname{grad} \operatorname{grad} \mid \mathbf{r}-\mathbf{r}_{(i)}^{\prime}\right) .
$$

The tensor $T_{2}$ also satisfies (1) but is regular in the region under consideration. We assume that $T_{2}$ may be determined explicitly for any particular surface $S_{2}$.

Now, proceeding formally as in the previous section, we obtain the same expression for $\mathbf{u}(P)$ as given by the relation (11) for any point $P$ in the region bounded by $S_{2}$ and $S_{1}$, with $S_{1}$ completely enclosed within $S_{2}$ :

$$
\mathbf{u}(P)=-\int_{S_{2}}\left[\left\{\mu \frac{d \mathbf{u}}{d n}+(\lambda+\mu) \theta \mathbf{n}\right\} \cdot \mathbf{T}-\mathbf{u} \cdot\left\{\mu \frac{d \mathbf{T}}{d n}+(\lambda+\mu) \theta \mathbf{n}\right\}\right] d S,
$$

where $P^{0}$ is a variable point on $S_{1}$ and, as before, $\mathrm{n}$ denotes the outward drawn normal to $S_{1}$. The additional condition of $\mathfrak{u}$ in the present case is that it vanishes on $S_{2}$. Moreover, when $S_{2}$ recedes to infinity, we rederive the result (11) by putting $T=T_{1}$. For the case when $\mathbf{u}$ is constant on $S_{1}$, we get the result similar to (12) with the total T:

$$
\mathbf{u}(P)=-\int_{S_{2}} \mathbf{f} \cdot \mathbf{T} d S=-\int_{S_{1}}\left\{\mu \frac{d \mathbf{u}}{d n}+(\lambda+\mu) \theta \mathbf{n}\right\} \cdot \mathbf{T} d S .
$$

The above formula is applicable to the problem of the displacement field generated by the translation of the light rigid particle $B$ which is given a uniform displacement $\mathrm{v}$ in the region bounded by the rigid boundary $S_{2}$. The formula (20) gives the required displacement field, while the formula 


$$
\mathbf{F}=\int_{S_{\mathbf{1}}} \mathbf{f} d S
$$

gives the corresponding static force $\mathbf{F}$ acting on $B$. Since we are going to relate this force with the one experienced by $B$ in an infinite elastic space, let us denote the static force $\mathbf{F}$ which $B$ would experience in an unbounded medium by $F_{\infty}$.

A precisely similar situation arises in slow viscous flow problems. There the concept of a resistance tensor is very handy. Analogously, we define a quantity which we shall call the traction tensor. For the case of an infinite elastic space we shall denote it as $\boldsymbol{\Phi}_{\infty}\left(\Phi_{\infty i j}\right)$. It has the property that the total static force exerted on a body which has been given a uniform translation $\mathbf{v}$ within an infinite elastic space has the value $-\left(d_{0} / a\right) \boldsymbol{\Phi}_{\infty} \cdot \mathrm{v}$. For the present case, when the elastic space is bounded by a rigid surface $S_{2}$, the corresponding traction tensor is $\boldsymbol{\Phi}\left(\Phi_{i j}\right)$ and the corresponding static force $\mathbf{F}$ is equivalent to $-\left(d_{0} / a\right) \mathbf{\Phi} \cdot \mathbf{v}$.

There arises a parameter $\epsilon$ in this problem. This is equal to the ratio of $a$, the characteristic dimension of $B$, to the minimum distance between a point of $S_{1}$ and a point of $S_{2}$. We take this quantity to be small in the present analysis. We further observe that $T$ and $T_{1}$ are both functions of $\mathbf{r}$ and $\mathbf{r}_{0}$ and since $T_{2}=T-T_{1}, T_{2}$ is also a function of $r$ and $\mathrm{r}_{0}$. If the origin 0 is taken to be some point of $S_{1}$ and $P$ and $P^{0}$ are also both on $S_{1}$, then it follows that

$$
\mathbf{T}_{2}=\mathbf{T}_{2}^{0}+\mathbf{r} \cdot\left[\operatorname{grad} \mathrm{T}_{2}\right]_{\mathrm{r}=\mathrm{r}_{0}=0}+\mathbf{r}_{0} \cdot\left[\operatorname{grad}^{0} T_{2}\right]_{\mathrm{r}=\mathrm{r}_{0}=0}+O\left(\epsilon^{3}\right),
$$

where $\mathbf{T}_{0}^{2}=\mathbf{T}_{2}(0,0)$ and the term $\operatorname{grad}^{0}$ implies the differentiation with respect to the components of $\mathbf{r}_{0}$.

The boundary value problem for the displacement field generated by the translation of $B$ gives rise to an integral equation similar to the one obtained in the previous section. In fact the corresponding formula in the present case is

$$
\frac{d_{0}}{a} \mathbf{I}=-\int_{S_{1}} \mathbf{f} \cdot\left(\mathbf{T}_{1}+\mathbf{T}_{2}\right) d S,
$$

with $P$ and $P^{0}$ both on $S_{1}$. If we neglect the contributions of the second and third terms on the right-hand side of Eq. (22) which are of $O\left(\epsilon^{2}\right)$, i.e., if we replace $T_{2}$ by $T_{2}^{0}$ in Eq. (23), it follows that

$$
\frac{d_{0}}{a} \mathrm{I}+\mathbf{F} \cdot \mathbf{T}_{2}^{0}=-\int_{S_{1}} \mathbf{f} \cdot \mathbf{T}_{1} d S
$$

This equation is the required integral equation for the displacement vector in the space enclosed between $S_{2}$ and $S_{1}$. But this same integral equation can be considered to give the displacement vector in an unbounded medium with $B$ having been given the uniform displacement $\left(d_{0} / a\right) \mathbf{I}+\mathbf{F} \cdot \mathbf{T}_{2}^{0}$. Therefore Eqs. (21) and (24) give

$$
\mathrm{F}=-\left(\frac{d_{0}}{a} \mathrm{I}+\mathrm{F} \cdot \mathrm{T}_{2}^{0}\right) \cdot \boldsymbol{\Phi}_{\mathrm{\infty}},
$$

and the solution of Eq. (25) is

$$
\mathbf{F}=-\frac{d_{0}}{a} \mathbf{I} \cdot\left[\boldsymbol{\Phi}_{\infty}^{-1}+\mathrm{T}_{2}^{0}\right]^{-1} .
$$


Furthermore, it readily follows from Eq. (25) that if $\mathbf{F}$ on the right-hand side is replaced by $F_{\infty}$, then the formula becomes

$$
\mathbf{F} \cong-\left(\frac{d_{0}}{a} \mathbf{I}+F_{\infty} \cdot T_{2}^{0}\right) \cdot \Phi_{\infty},
$$

and the error in Eq. (27) is of order $O\left(\epsilon^{2}\right)$.

We now consider the simplified case when $I$ is parallel to one of the principal axes of the tractions on $S_{1}$; these axes being defined so that, in translation through an unbounded medium parallel to one of them, the total static force on $B$ is in the direction of the translation. In this case Eq. (25) takes a simple form. In fact, let $\mathbf{e}_{1}, \mathbf{e}_{2}, \mathbf{e}_{3}$ stand for the unit eigenvectors of $\boldsymbol{\Phi}_{\infty}$ such that

$$
\boldsymbol{\Phi}_{\infty}=\Phi_{\infty 1} \mathbf{e}_{1} \mathbf{e}_{2}+\Phi_{\infty 2} \mathbf{e}_{2} \mathbf{e}_{2}+\Phi_{\infty 3} \mathbf{e}_{3} \mathbf{e}_{3},
$$

and decompose the tensor $\mathrm{T}_{2}^{0}$ into the components $T_{2 i j}^{0}$ with respect to these three unit vectors as the basis. Also let $\mathbf{I}=\mathbf{e}_{1}$. Substituting these expressions in Eq. (25), we derive the formula

$$
F / F_{\infty}=1 /\left(1-\lambda F_{\infty}\right),
$$

where $\lambda$ is independent of the form of $S_{1}$. Similarly, when $B$ is moving parallel to a principal axis of the traction, it follows from Eq. (27) that

$$
F / F_{\infty} \simeq 1+\lambda F_{\infty},
$$

where $\lambda F_{\infty}$ is $O(\epsilon)$; hence, neglecting the term $O\left(\epsilon^{2}\right)$, Eqs. (29) and (30) are identical, as are Eq. (26) and (27).

4. Elastodynamics. We have recently derived formulas [12] which give the dynamical displacement field in an infinite elastic space wherein is embedded a light rigid axially symmetric particle. This particle is excited by a periodic force acting in the direction of its axis of symmetry. In this section we discuss the generalization of that problem for a particle of an arbitrary shape. This particle is depressed by an amount $d \mathbf{I}=d_{0} e^{i \omega t} \mathbf{I}$, where $\mathbf{I}$ is a unit vector, into the unbounded medium by an exciting force of the same frequency $\omega$.

In the equations of elastodynamics,

$$
(\lambda+\mu) \operatorname{grad} \theta+\mu \nabla^{2} \mathbf{u}-\rho \partial^{2} u / \partial t^{2}=0,
$$

we set $\mathfrak{u}=\mathfrak{u}_{0} e^{i \omega t}$ and drop the zero subscript, obtaining thereby the equation

$$
(\lambda+\mu) \operatorname{grad} \theta+\mu \nabla^{2} \mathbf{u}+\rho \omega^{2} \mathbf{u}=0,
$$

where $\rho$ is the density of the medium. If we now nondimensionalize this equation by the help of the characteristic length $a$ of the body, it takes the form

$$
(\lambda+\mu) \operatorname{grad} \theta+\mu \nabla^{2} \mathbf{u}+\rho \omega^{2} a^{2} \mathfrak{u}=0 .
$$

In our previous work [12] on axially symmetric bodies, we defined two dimensionless parameters:

$$
M^{2}=\rho \omega^{2} a^{2} /(\lambda+2 \mu) ; \quad m^{2}=\rho \omega^{2} a^{2} / \mu .
$$

Furthermore, a material constant $\tau$ was defined as

$$
\tau=M / m=(\mu)^{1 / 2} /(\lambda+2 \mu) .
$$

These quantities are helpful in the present analysis as well. 
The next step is to derive an appropriate integral representation for $\mathfrak{u}$. In fact, if we repeat the analysis as given in Sec. 2 for the case of static elasticity with present differential equations, we find that the value of $\mathbf{u}$ at an arbitrary point $P$ in the medium exterior to $S$ is given by the same expression:

$$
\mathbf{u}=-\int_{S} \mathbf{f} \cdot \mathbf{T}-\mathbf{u} \cdot\left(\mu \frac{d \mathbf{T}}{d n}+(\lambda+\mu) \theta \mathbf{n}\right) d S,
$$

where $\mathbf{f}$, as before, is equal to $\mu d \mathfrak{u} / d n+(\lambda+\mu) \theta \mathbf{n}$. The tensor $\mathbf{T}$ is now the Green's tensor for Eq. (33) and we assume that it has the expression

or

$$
\mathrm{T}=\mathrm{U}\left(\nabla^{2}+M^{2}\right) \phi-\frac{\lambda+\mu}{\lambda+2 \mu} \operatorname{grad} \operatorname{grad} \phi,
$$

$$
T_{i j}=\delta_{i j}\left(\nabla^{2}+M M^{2}\right) \phi-\frac{\lambda+\mu}{\lambda+2 \mu} \frac{\partial^{2} \phi}{\partial x_{i} \partial x_{j}} .
$$

The function $\phi$ satisfies the differential equation

$$
\left(\nabla^{2}+m^{2}\right)\left(\nabla^{2}+M^{2}\right) \phi=0 .
$$

An appropriate solution of Eq. (38) is

$$
\phi=\frac{1}{4 \pi} \frac{1}{\left(m^{2}-M^{2}\right)}\left[\frac{\exp \left(-i M\left|\mathbf{r}-\mathbf{r}_{0}\right|\right)}{\left|\mathbf{r}-\mathbf{r}_{0}\right|}-\frac{\exp \left(-i m\left|\mathbf{r}-\mathbf{r}_{0}\right|\right)}{\left|\mathbf{r}-\mathbf{r}_{0}\right|}\right] .
$$

Let us note that the parameters $M$ and $m$ are of the same order of magnitude because their ratio $\tau$ is just a material constant. When we substitute (39) in (37) and then the resulting value of $\mathbf{T}$ in (36), we observe that $\mathbf{u}$ is a complex quantity. Furthermore, if we expand this value of $\mathbf{T}$ in powers of $m$, we get

$$
\mathrm{T}=\mathbf{T}_{0}-\frac{i}{12 \pi}\left(\tau^{3}+2\right) m \mathbf{U}+O\left(m^{2}\right),
$$

where $\mathbf{T}_{0}$, as derived in Sec. 2 , is

$$
\mathbf{T}_{0}=\frac{1}{8 \pi}\left\{U \nabla^{2}\left|\mathbf{r}-\mathbf{r}_{0}\right|-\frac{\lambda+\mu}{\lambda+2 \mu} \operatorname{grad} \operatorname{grad}\left|\mathbf{r}-\mathbf{r}_{0}\right|\right\} .
$$

The elastodynamic boundary value problems can now be solved in the same fashion as the previous elastostatic problems. Let us first observe that the boundary conditions are $\mathfrak{u}=\left(d_{0} / a\right) I$ on $S$ and $\mathfrak{u}$ satisfies the radiation condition at $\infty$. The integral equation governing the displacement field is obtained by taking $P$ in Eq. (36) to be on $S$ and setting $\mathbf{u}=\left(d_{0} / a\right)$ I on $S$. As $\mathbf{u}$ is constant on $S$, it may be taken outside the integral in Eq. (36), It now follows from the relation

$$
-m^{2} \mathbf{T}=(\lambda+\mu) \operatorname{grad} \boldsymbol{\theta}+\mu \nabla^{2} \mathbf{T}
$$

and Green's theorems that

$$
\int_{S}\left(\mu \frac{d \mathbf{T}}{d n}+(\lambda+\mu) \theta \mathbf{n}\right) d S=-m^{2} \int_{0} \mathbf{T} d V,
$$

where $V$ denotes the interior of $B$. Recalling the formula for the total force $\mathrm{F}$,

$$
\mathbf{F}=\int_{S} \mathbf{f} d S,
$$


we readily obtain from the relations (36), (40), and (43) the result

$$
\frac{d_{0}}{a} \mathbf{I}-\frac{i}{12 \pi}\left(\tau^{3}+2\right) m \mathbf{F}=-\int_{S} \mathbf{T}_{0} \cdot \mathbf{f} d S+O\left(m^{2}\right) \text {. }
$$

When $m=0$, the above equation reduces to Eq. (17).

By following the same steps as we did in deriving relation (27), we get the corresponding force formula as

$$
\mathbf{F}=\boldsymbol{\Phi}_{\infty} \cdot\left\{\frac{d_{0}}{a} \mathbf{I}-\frac{i}{12 \pi}\left(\tau^{3}+2\right) m\left(-\frac{d_{0}}{a} \boldsymbol{\Phi}_{\infty} \cdot \mathbf{I}\right)\right\}+O\left(m^{2}\right) .
$$

For a body displaced parallel to one of its principal axes of traction which, as defined before, are the directions such that $-\left(d_{0} / a\right) \mathbf{\Phi}_{\infty} \cdot \mathbf{u}=\mathbf{F}_{\infty}=-F_{\infty} \mathbf{u}$, Eq. (46) takes the simple form

$$
\mathbf{F}=-F_{\infty}\left\{1+\left(a / d_{0}\right)\left(F_{\infty} i / 12 \pi\right)\left(\tau^{3}+2\right) m\right\} \mathrm{I}+O\left(m^{2}\right) .
$$

If we set $F_{\infty}=X_{0} / \mu a^{2}$, where $X_{0}$ is given in the physical units, the formula (47) reduces to the one derived for the axially symmetric bodies by us [12]. In our previous work we had mistakenly ${ }^{1}$ assumed that the result (47) is applicable also to nonaxially symmetric configurations.

As an application of the general formula (47), we consider the case of a thin rigid circular disk of radius $a$ attached to the free surface of an elastic half space and excited by a periodic force of frequency $\omega$ acting at angle $\alpha$ to its axis of symmetry. Let $\mathbf{e}_{1}$ be a unit vector in the plane of the disk and let $\mathbf{e}_{2}$ be unit vector along the axis of symmetry. The force formula for a translation of amount $d_{0}$ of the disk along the directions $\mathbf{e}_{2}$ is known and when written in physical units is [15]

$$
X_{0}=8 \pi \mu d_{0}\left(1-\tau^{2}\right) a .
$$

Similarly, the static force for a uniform translation of amount $d_{0}$ along the direction $\mathbf{e}_{1}$ is

$$
X_{0}=16 \pi \mu d_{0}\left(\tau^{2}+3\right)^{-1} a .
$$

When these formulas are substituted in Eq. (46), we derive the required force in physical units:

$$
\begin{aligned}
\mathrm{X}=-\pi \mu a d_{1} e^{i \omega t}[8 & \left(1-\tau^{2}\right) \cos \alpha \mathrm{e}_{2}\left\{1+(2 i / 3 \pi)\left(1-\tau^{2}\right)\left(\tau^{3}+2\right) m\right\} \\
& \left.+16\left(\tau^{2}+3\right)^{-1} \sin \alpha \mathrm{e}_{1}\left\{1+(4 i / 3 \pi)\left(\tau^{2}+3\right)^{-1}\left(\tau^{3}+2\right) m\right\}\right] .
\end{aligned}
$$

Acknowledgment. This research was sponsored by a grant from the National Science Foundation to the Pennsylvania State University.

\section{References}

[1] E. Betti, I1 Nuovo Cimento 6-10 (1872)

[2] C. Somigliana, I1 Nuovo Cimento 17-20 (1885); Annali di Matematica (2) 17 (1889)

[3] I. Fredholm, Solution d'un problème fondamental de la théorie de l'élasticité, Arch. Mat. Astronom. Fysik, 2 (1905)

[4] G. Lauricella, Sur l'integration de l'équation relative à l'equilibre des plaques élastiques encastrées, Acta Math. 32 (1909)

'Note added in proof: The author's attention has been drawn to the paper by W. E. Williams, Quart. J. Mech. Appl. Math. 19, 414 (1966), wherein this error in his previous work is pointed out. 
[5] D. I. Sherman, Dokl. Akad. Nauk SSSR 27-28 (1940); 32 (1941)

[6] N. I. Muskhelishvili, Some basic problems of the mathematical theory of elasticity, Noordhoff, Holland, 1953

[7] S. G. Mikhlin, Integral equations, Pergamon Press, London, 1957

[8] M. A. Jaswon and A. R. Ponter, An integral equation solution of the torsion problem, Proc. Roy Soc. Series A, 273 (1963)

[9] F. J. Rizzo, An integral equation approach to boundary value problems of classical elastostatics, Quart. Appl. Math. 25 (1967)

[10] C. W. Oseen, Neuere Methoden und Ergebnisse in der Hydrodynamik, Leipzig, 1927

[11] W. E. Williams, Boundary effects in Stokes flow, J. Fluid Mech. 24 (1966)

[12] R. P. Kanwal, Dynamical displacements in an infinite elastic space and matched asymptotic expansions, J. Math. Phys. 44 (1965). Errata ibid. 47 (1965)

[13] J. B. Diaz and H. J. Greenberg, Upper and lower bounds for the solution of the first boundary value problems of elasticity, Quart. Appl. Math. 6 (1948)

[14] A. E. H. Love, Mathematical theory of elasticity, 4th ed., Dover, New York, 1944

[15] G. N. Bycroft, Forced vibrations of a rigid circular plate on a semi-infinite elastic space on an elastic stratum, Trans. Royal Philos. Soc., 248 (1955-1956)

[16] V. D. Kupradze, Dynamical problems in elasticity, North-Holland Publishing Company, Amsterdam, 196:3 